\title{
THE
}

6-8-2015

\section{Multiplexed Displacement Fiber Sensor Using Thin Core Fiber Exciter}

Zhen Chen

University of Rhode Island

Gerald Hefferman

Tao Wei

University of Rhode Island, tao_wei@uri.edu

Follow this and additional works at: https://digitalcommons.uri.edu/ele_facpubs

Terms of Use

All rights reserved under copyright.

\section{Citation/Publisher Attribution}

Zhen Chen, Gerald Hefferman and Tao Wei. (2015). Multiplexed displacement fiber sensor using thin core fiber exciter. Review of Scientific Instruments, 86(6), 065004. doi: 10.1063/1.4922019

Available at: http://dx.doi.org/10.1063/1.4922019

This Article is brought to you for free and open access by the Department of Electrical, Computer, and Biomedical Engineering at DigitalCommons@URI. It has been accepted for inclusion in Department of Electrical, Computer, and Biomedical Engineering Faculty Publications by an authorized administrator of DigitalCommons@URI. For more information, please contact digitalcommons-group@uri.edu. 


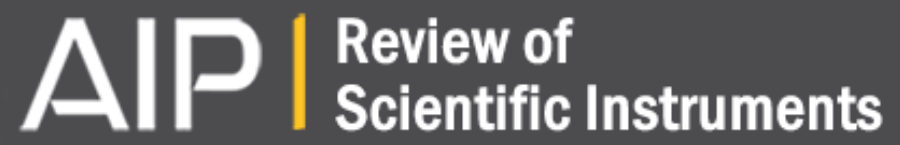

\section{Multiplexed displacement fiber sensor using thin core fiber exciter}

Zhen Chen, Gerald Hefferman, and Tao Wei

Citation: Review of Scientific Instruments 86, 065004 (2015); doi: 10.1063/1.4922019

View online: http://dx.doi.org/10.1063/1.4922019

View Table of Contents: http://scitation.aip.org/content/aip/journal/rsi/86/6?ver=pdfcov

Published by the AIP Publishing

\section{Articles you may be interested in}

Multiplex and simultaneous measurement of displacement and temperature using tapered fiber and fiber Bragg grating

Rev. Sci. Instrum. 83, 053109 (2012); 10.1063/1.4718360

Long distance fiber-optic displacement sensor based on fiber collimator

Rev. Sci. Instrum. 81, 123104 (2010); 10.1063/1.3518971

Fiber optic displacement sensor using a coated lens optic

Rev. Sci. Instrum. 71, 4314 (2000); 10.1063/1.1290498

A frequency division multiplexed low-finesse fiber optic Fabry-Perot sensor system for strain and displacement measurements

Rev. Sci. Instrum. 71, 1275 (2000); 10.1063/1.1150453

Design and operation of different optical fiber sensors for displacement measurements

Rev. Sci. Instrum. 70, 2875 (1999); 10.1063/1.1149812

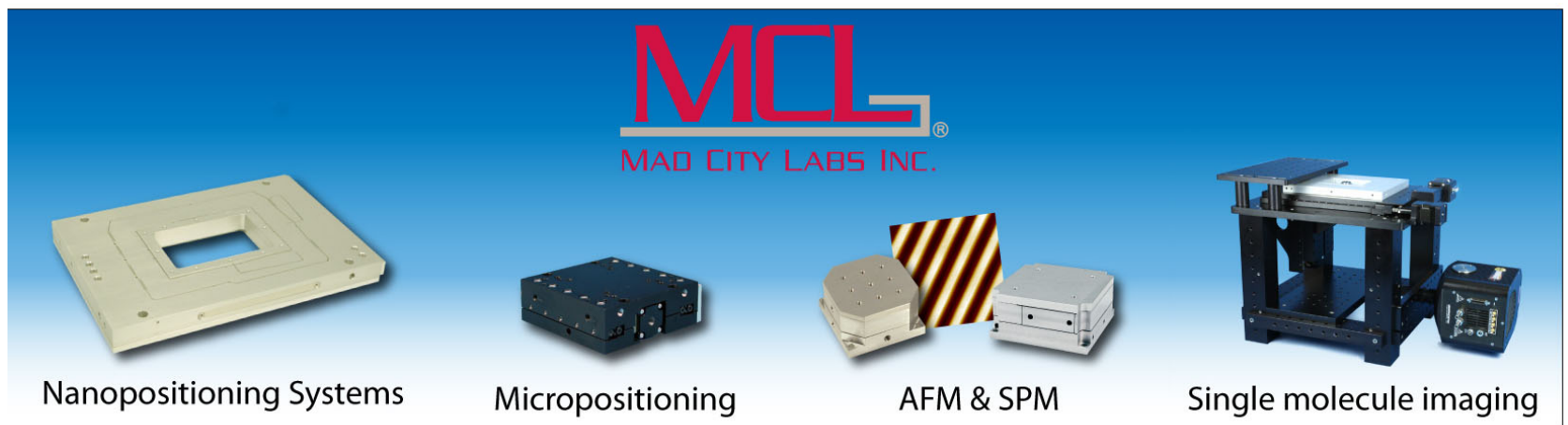




\title{
Multiplexed displacement fiber sensor using thin core fiber exciter
}

\author{
Zhen Chen, ${ }^{1}$ Gerald Hefferman, ${ }^{1,2}$ and Tao Wei ${ }^{1, a)}$ \\ ${ }^{1}$ Department of Electrical, Computer and Biomedical Engineering, University of Rhode Island, Kingston, \\ Rhode Island 02881, USA \\ ${ }^{2}$ Warren Alpert Medical School of Brown University, Providence, Rhode Island 02903, USA
}

(Received 9 April 2015; accepted 21 May 2015; published online 8 June 2015)

\begin{abstract}
This letter reports a multiplexed optical displacement sensor using a thin core fiber (TCF) exciter. The TCF exciter is followed by a stripped single mode optical fiber. A small section of buffer is used as the movable component along the single mode fiber. Ultra-weak cladding mode reflection $(<-75 \mathrm{~dB})$ was employed to probe the refractive index discontinuity between the air and buffer coating boundary. The position change of the movable buffer segment results in a delay change of the cladding mode reflection. Thus, it is a measure of the displacement of the buffer segment with respect to the glass fiber. The insertion loss of one sensor was measured to be less than $3 \mathrm{~dB}$. A linear relationship was evaluated between the measurement position and absolute position of the moving actuator. Multiplexed capability was demonstrated and no cross talk was found between the sensors. (C) 2015 AIP Publishing LLC. [http://dx.doi.org/10.1063/1.4922019]
\end{abstract}

\section{INTRODUCTION}

Fiber optic displacement sensors have several distinct advantages that make them particularly advantageous as compared to conventional capacitive and inductive displacement sensors, including compact size, thermal and chemical resilience, multiplexing capacity, and electromagnetic immunity. These attributes have led to the successful application of optical fiber sensors in structural monitoring and machine control. ${ }^{1,2}$ Fiber grating-based sensors, including fiber Bragg gratings (FBGs), ${ }^{3-5}$ long period fiber gratings (LPFGs), ${ }^{6,7}$ and tilted fiber Bragg gratings (TFBG), ${ }^{8}$ have been employed as displacement measurement devices, either by direct stretch, the use of cantilever beams and similar structures, or perpendicular displacement to the sensors. Unfortunately, the abovementioned displacement sensing technologies have a major limitation that the measurement dynamic range is restricted due to the glass nature of optical fiber. A loaded strain of more than $0.4 \%$ can break the fiber and immediately fail the sensor. In addition, the high signal strength of the existing fiber optic displacement sensors restricts them to be used in a multiplexed fashion. In order to fundamentally tackle these limitations, a new fiber optic based displacement sensing concept with large dynamic range and small signal strength is necessary.

Evanescent wave of cladding mode of an optical fiber is potentially a feasible probing solution, since it extends the light energy from the core of optical fiber to the ambience, removing the need to stretch fiber glass to sense displacement, allowing for a much larger dynamic range. In research community, this sensing approach was widely used to probe ambient change of optical fiber for various physical and chemical sensing applications. ${ }^{9-16}$ A recent alternative to conventional displacement sensing approaches is the use of a fiber modal interferometer, where the optical path difference between the basic core

\footnotetext{
a) Author to whom correspondence should be addressed. Electronic mail: tao_wei@uri.edu.
}

mode and excited cladding modes is measured. These fiber modal interferometers for displacement sensing have been successfully constructed using thin core fiber (TCF), ${ }^{17}$ thermal expended fiber (TEF), ${ }^{18}$ and photonic crystal fiber (PCF), ${ }^{19}$ where displacement is introduced by direct strain on the fiber under test. Each of these sensors has resulted in high accuracy measurements and improved dynamic range. However, each of these sensors requires a relative large portion of the total light energy for sensing, limiting its potential to be multiplexed along one fiber line. It is worth noting that the cost of fiber optic sensor majorly comes from the interrogation instrument. By multiplexing a large number of fiber optic sensors along a single fiber line, one can effectively reduce the cost of each sensor and widen its broad adoption.

This letter reports a design of optical fiber device for displacement sensing that extends the use of these weakly coupled evanescent waves. The resulting sensor is inexpensive and simple to fabricate. TCF was used to excite and receive cladding modes used to probe the refractive index discontinuity between the air and moving fiber buffer coating. Ultraweak cladding reflections $(<-75 \mathrm{~dB})$ were employed as a measure of buffer displacement along the length of the optical fiber under test. A linear relationship was found between the measurement result and absolute displacement values.

\section{OPERATION MECHANISM}

In order to probe the optical fiber under test, optical frequency domain reflectometry (OFDR) was used as an interrogation mechanism..$^{20-22}$ The detection bandwidth is setup by the scanning range of the tunable laser, which can be calculated by

$$
\mathrm{B}=\frac{\mathrm{c}}{\lambda^{2}} \Delta \lambda,
$$

where $\mathrm{c}$ is the speed of light in vacuum, $\lambda$ is the central wavelength, and $\Delta \lambda$ is wavelength sweep range. In this setup, illustrated in Fig. 1, the tunable laser sweeps from 1525 to 

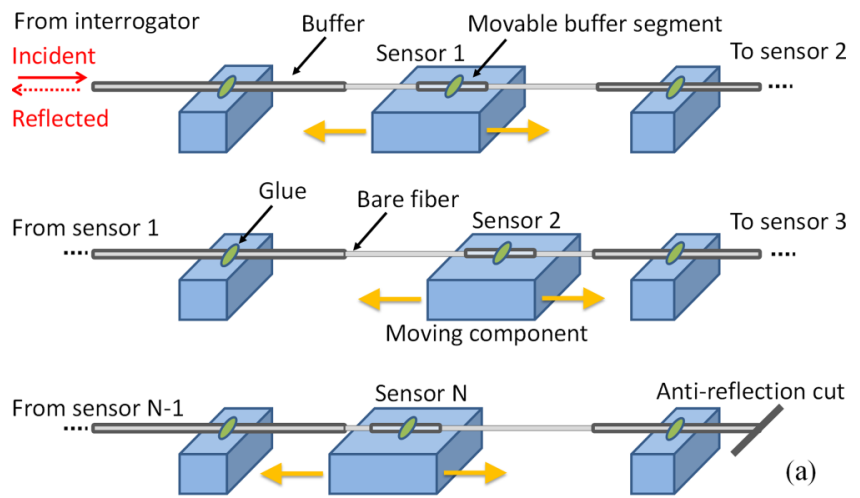

To multiplexed displacement sensors

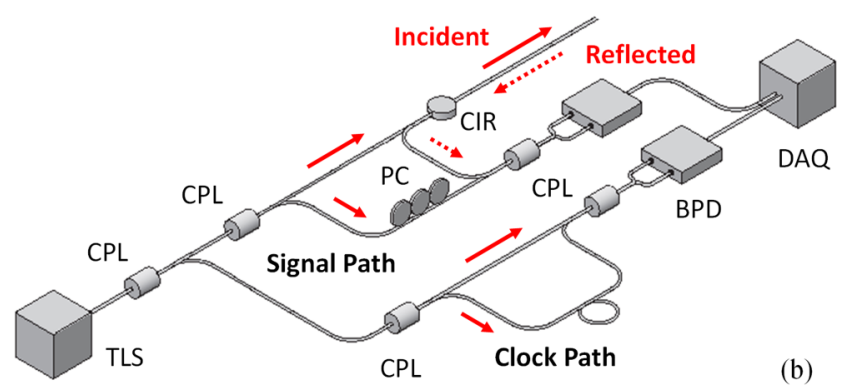

FIG. 1. Schematic of multiplexed displacement sensors: (a) the sensing array, (b) the interrogation system (TLS: tunable laser source, CPL: coupler, PC: polarization controller, CIR: circulator, BPD: balanced photodiode, DAQ: data acquisition card).

$1555 \mathrm{~nm}$ at $60 \mathrm{~nm} / \mathrm{s}$ scanning speed, corresponding to a total bandwidth of $3.8 \mathrm{THz}$. The real-time system has an update rate of $1.5 \mathrm{~s}$ per round, which includes $\sim 0.5 \mathrm{~s}$ for laser scanning and $\sim 1 \mathrm{~s}$ for transmission of the data to a polarization controller (PC) and calculation. The sensor was fabricated by fusionsplicing a short segment (sub-centimeter) of TCF (Nufern, PSGSF-3/125, core diameter $3 \mu \mathrm{m}$, cladding diameter $125 \mu \mathrm{m}$ ) within a single mode fiber (SMF) segment (Corning SMF$28 \mathrm{e}$, core diameter $8.2 \mu \mathrm{m}$, cladding diameter $125 \mu \mathrm{m}$ ) using a conventional SMF splice program, illustrated in Fig. 2(a). The core diameter mismatch between the TCF and SMF introduces a small portion of optical energy from the core mode into the cladding modes, functionally converting the TCF to a cladding mode exciter. The effective refractive indices (RIs) of these cladding modes are determined by the boundary condition at the interface between fiber cladding and surrounding material. A short segment of buffer $(\sim 1 \mathrm{~mm})$ was carefully separated from the sensing SMF using a Ripley Miller fiber optic stripper but remained intact and moveable along the fiber cladding, shown in Fig. 2(d). Due to the elastic property of polymer coating, the buffer segment is in close contact with the bare fiber. To investigate the stability of the proposed sensor, a test was performed in which the buffer remained unchanged. Hundred waveforms were recorded using the fixed configuration, resulting in a calculated stability of $54 \mu \mathrm{m}$. The SMF-28e buffer coating is composed of dual acrylate with a refractive index about 1.48 at $1550 \mathrm{~nm}$. In this experiment, the buffer was attached to an automated precision motion stage that moved along the fiber direction. When the forward propagating cladding modes meet the buffer-air boundary, the surrounding

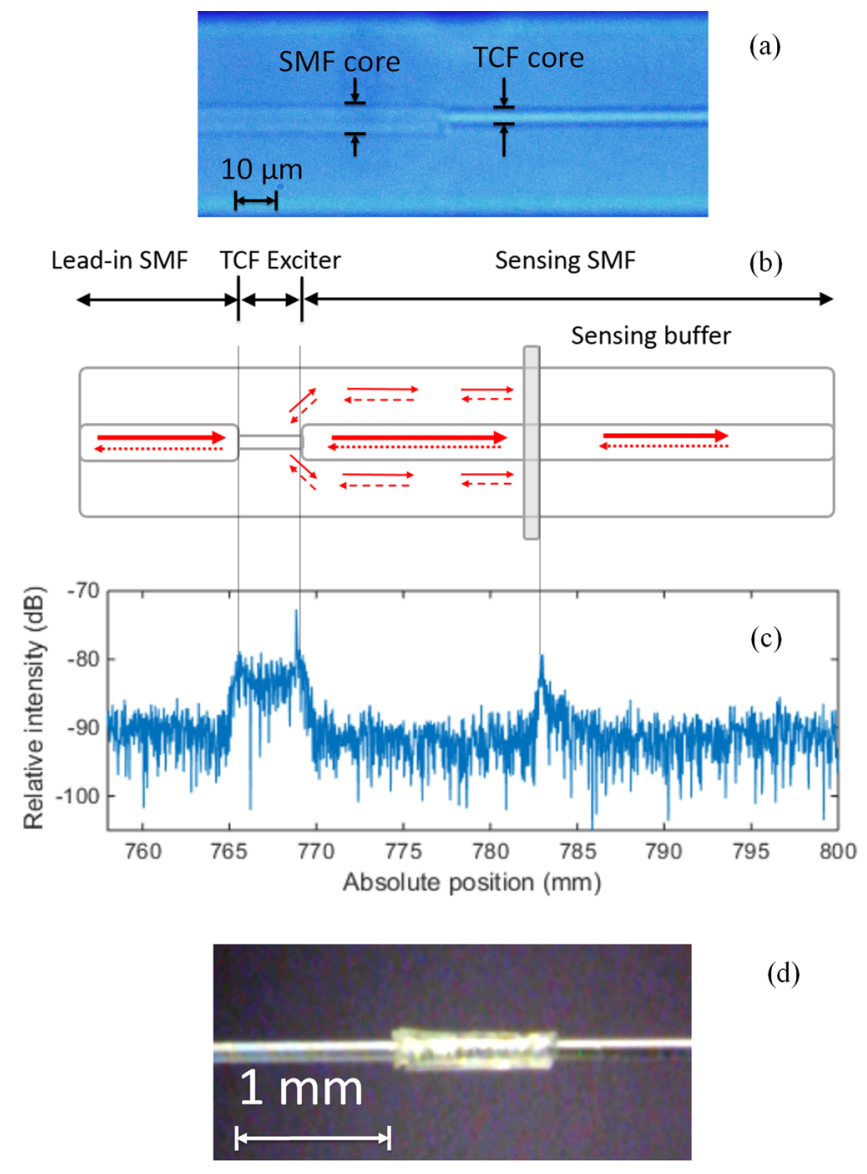

FIG. 2. The proposed displacement sensor: (a) microscopic image of the core-mismatch between the SMF and TCF, (b) diagram of operation mechanism, (c) signal of a time domain reflection peak from the experiment, and (d) image of buffer segment.

RI discontinuity leads to a cladding mode reflection. These back-propagating cladding reflections then couple back to the leading SMF via the TCF exciter, resulting a cladding mode reflection peak in distance domain as pictured in Fig. 2(c). The TCF exciter has a higher Rayleigh level as compared to the neighboring SMF. As the stage and the attached buffer move along the fiber, a resulting reflection peak position displace-
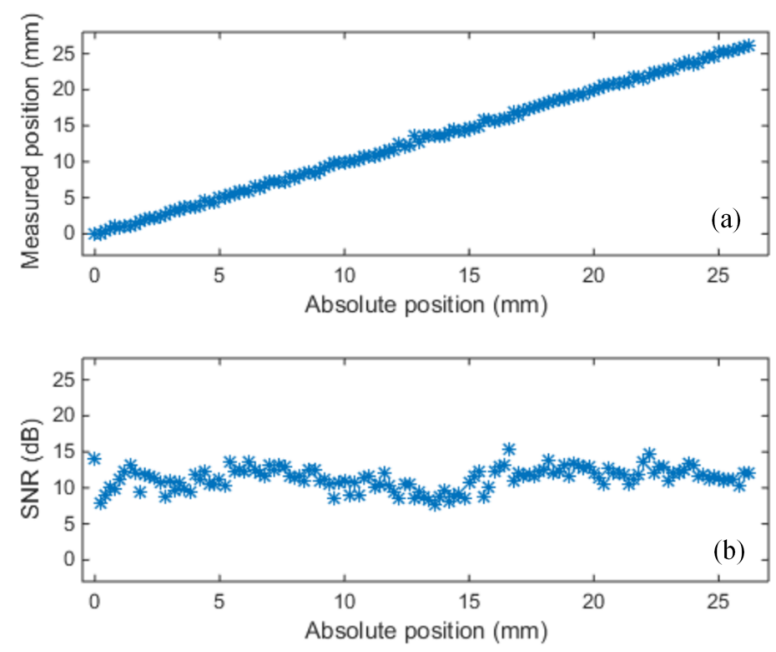

FIG. 3. Displacement measurement: (a) measured position against absolute position and (b) SNR as a function of absolute position. 

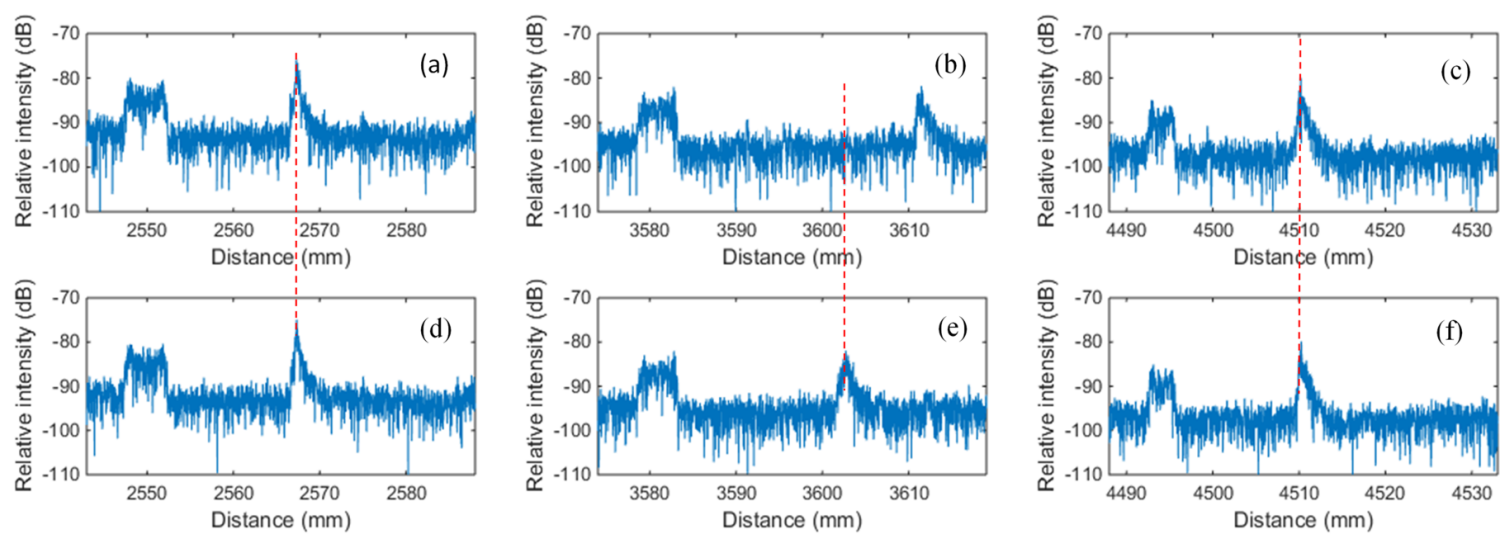

FIG. 4. Multiplexed sensing test with three TCF exciter displacement sensors in-line: (a) and (d) was stable sensor 1; (b) and (e) was shifted sensor 2; (c) and (f) was shifted sensor 3 .

ment can be measured using the previously described OFDR system.

\section{EXPERIMENTAL RESULTS}

To investigate the utility of the proposed device, a TCF displacement sensor was fabricated with a TCF length of $5 \mathrm{~mm}$. The insertion loss of this sensor is less than $3 \mathrm{~dB}$. The buffer was attached to an actuator (Newport PM500 series, maximum range $26.4 \mathrm{~mm}$, resolution $100 \mathrm{~nm}$ ) moving along the fiber direction in $0.2 \mathrm{~mm}$ increments over a total distance of $26.4 \mathrm{~mm}$. In Fig. 3(a), the measurement result was plotted with the absolute position read from the stage controller. After applying a linear fit to the experimental data, the calculated $\mathrm{R}^{2}$ value was $99.92 \%$, indicating a highly linear relationship between the measured position and absolute position. The signal-to-noise ratio (SNR) of the detection peak was examined in Fig. 3(b), where the signal was defined as the relative intensity of the reflection peak and the noise was by averaging the Rayleigh backscatter relative intensity level over $10 \mathrm{~mm}$ in the sensing region. The standard deviation of the measurement was found to be $0.2134 \mathrm{~mm}$. The average SNR was $13.1 \mathrm{~dB}$ over the detection region. It also indicates that the reflection coefficient from the buffer segment is sufficiently stable in practice.

To demonstrate the multiplexing capability of the proposed device, 3 sensors ( $5 \mathrm{~mm}$ TCF) were identically fabricated and spliced in-line along the interrogation system. Each sensor was about 1 meter apart. All sensors had stripped buffers which could move along the fiber under test. Displacement was tested using the second sensor, while the first and third sensors remained stationary. Figs. 4(b) and 4(e) show the second sensor signal peak shift due to the buffer displacement, while the first peak signal in Figs. 4(a) and 4(d) and the third peak signal in Figs. 4(c) and 4(f) remained stable. The major cross coupling is rooted from the multiple-reflection cross talk, or the signal waveform distortion induced by "ghost" signals, which undergo multiple reflections between upstream sensors, and experiences the same time delay as the real signal. The multiple-reflection noise has been thoroughly studied in previous weak-reflection sensing studies. ${ }^{23}$ Accordingly, a computer simulation based on the previous model was conducted to theoretically investigate the noise that solely contributed by the multiple reflections. This simulation suggests that the SNR remains above $55 \mathrm{~dB}$, as the number of multiplexed sensors increases to $100 \mathrm{~K}$. Thus, the multiple-reflection cross talk can be ignored since it is far below the background noise of the system. Shown from Fig. 4, no cross talk noise was observed in our experiment.

Potentially, this technology can be used to provide feedback measurement of automation system. It is especially attractive when more than one linear movement is needed to be monitored in the system on a real-time basis. The limitation of this technology is the contamination of the cladding surface of the bare fiber. The accumulated dirt may result in fake signals. In order to prevent contamination, engineering efforts are necessary to properly package each of the sensor head.

\section{CONCLUSION}

To conclude, this letter reports a design of a fiber displacement sensor using a TCF as a cladding mode exciter. Ultraweak cladding mode reflections $(<-75 \mathrm{~dB})$ were employed to probe the external refractive index change at the air-buffer boundary. A linear relationship was evaluated between the measurement position and the absolute position during the displacement test. Multiplexing capability was demonstrated and no cross talk was found between each sensor.

${ }^{1}$ Y. Huang, T. Wei, Z. Zhou, Y. Zhang, G. Chen, and H. Xiao, Meas. Sci. Technol. 21, 105308 (2010).

${ }^{2}$ R. Tennyson, A. Mufti, S. Rizkalla, G. Tadros, and B. Benmokrane, Smart Mater. Struct. 10, 560 (2001).

${ }^{3}$ Y. Zhu, P. Shum, C. Lu, M. Lacquet, P. Swart, A. Chtcherbakov, and S. Spammer, Opt. Express 11, 1918 (2003).

${ }^{4}$ Y. Zhao, H. Huang, and Q. Wang, Sens. Actuators, A 165, 185 (2011).

${ }^{5}$ J. H. Ng, X. Zhou, X. Yang, and J. Hao, Opt. Commun. 273, 398 (2007).

${ }^{6}$ G. Chen, H. Xiao, Y. Huang, Y. Zhang, and Z. Zhou, "Simultaneous strain and temperature measurement using long-period fiber grating sensors," Proc. SPIE 7649, 764902 (2010).

${ }^{7}$ M. Han, F. Guo, and Y. Lu, Opt. Lett. 35, 399 (2010).

${ }^{8}$ Q. Jiang and D. Hu, IEEE Sens. J. 11, 1776 (2011).

9 J. Huang, X. Lan, H. Wang, L. Yuan, T. Wei, Z. Gao, and H. Xiao, Opt. Lett. 37, 4308 (2012).

${ }^{10}$ Q. Han, X. Lan, J. Huang, A. Kaur, T. Wei, Z. Gao, and H. Xiao, IEEE Photonics Technol. Lett. 24, 1130 (2012).

${ }^{11}$ J. Huang, X. Lan, A. Kaur, H. Wang, L. Yuan, and H. Xiao, Opt. Eng. 52, 014404 (2013).

${ }^{12}$ X. Lan, B. Cheng, Q. Yang, J. Huang, H. Wang, Y. Ma, H. Shi, and H. Xiao, Sens. Actuators, B 193, 95 (2014). 
${ }^{13}$ J. Huang, X. Lan, A. Kaur, H. Wang, L. Yuan, and H. Xiao, Sens. Actuators, B 198, 384 (2014).

${ }^{14}$ X. Wen, J. Huang, H. Xiao, and M. Yang, Meas. Sci. Technol. 25, 114002 (2014).

${ }^{15}$ X. Lan, J. Huang, Q. Han, T. Wei, Z. Gao, H. Jiang, J. Dong, and H. Xiao, Opt. Lett. 37, 1998 (2012).

${ }^{16}$ J. Zhang, X. Tang, J. Dong, T. Wei, and H. Xiao, Sens. Actuators, B 135, 420 (2009).

${ }^{17}$ J. Wu, Y. Miao, B. Song, W. Lin, K. Zhang, H. Zhang, B. Liu, and J. Yao, Opt. Commun. 340, 136 (2015).
${ }^{18}$ A. Sun and Z. Wu, Opt. Commun. 348, 50 (2015).

${ }^{19}$ J. N. Dash, R. Jha, J. Villatoro, and S. Dass, Opt. Lett. 40, 467 (2015).

${ }^{20}$ Z. Chen, L. Yuan, G. Hefferman, and T. Wei, Opt. Lett. 40, 320 (2015).

${ }^{21}$ Z. Chen, L. Yuan, G. Hefferman, and T. Wei, IEEE Photonics Technol. Lett. 27, 1084 (2015).

${ }^{22}$ Z. Chen, Y. Zeng, G. Hefferman, Y. Sun, and T. Wei, in Proceedings of IEEE Workshop on Information Forensics and Security (WIFS'14), Altalanta, GA, 3-5 December 2014.

${ }^{23}$ Y. Wang, J. Gong, B. Dong, D. Y. Wang, T. J. Shillig, and A. Wang, J. Lightwave Technol. 30, 2751 (2012). 